Research Paper

\title{
Superior survival benefits of Radical Prostatectomy than External Beam Radiotherapy in aging 75 and older men with high-risk or very high-risk Prostate Cancer: a population-matched study
}

\author{
Yan Wang ${ }^{1 \star *}$, Pan Song ${ }^{2 *}$, Jiaxiang Wang ${ }^{3}$, Mengxuan Shu ${ }^{3}$, Qingwei Wang ${ }^{1}$, Qi Li ${ }^{1}$ \\ 1. Department of Urology, The First Affiliated Hospital of Zhengzhou University, Zhengzhou, 450052, Henan Province, China. \\ 2. Department of Urology, West China Hospital of Sichuan University, Chengdu, 610000, Sichuan Province, China. \\ 3. The first Clinical Medical College of Lanzhou University, Lanzhou, 730000, Gansu Province, China. \\ ${ }^{*}$ Equal contributions to this work \\ $\triangle$ Corresponding author: Yan Wang. Department of Urology, The First Affiliated Hospital of Zhengzhou University, Jianshe road No. 1, Zhengzhou city, \\ 450000, China. E-mail: yanwangzzu@163.com.
}

(1) The author(s). This is an open access article distributed under the terms of the Creative Commons Attribution License (https://creativecommons.org/licenses/by/4.0/). See http://ivyspring.com/terms for full terms and conditions.

Received: 2020.03.16; Accepted: 2020.06.29; Published: 2020.07.11

\begin{abstract}
Objective: To evaluate the survival difference of radical prostatectomy (RP) and external beam radiotherapy (EBRT) in elderly men (75 years and older) with high-risk (HR) or very high-risk (VHR) prostate cancer (PCa).

Methods: Elderly men diagnosed with HR/VHR PCa from 2004-2015 in the Surveillance, Epidemiology and End Results (SEER) database were identified. Propensity-score matching (PSM) was conducted to balance the covariates; Kaplan-Meier and Cox analysis were performed to evaluate the overall survival (OS) and prostate cancer-specific survival (PCSS).

Results: 11698 patients with HR PCa and 4415 patients with VHR PCa were identified and divided into RP and EBRT group. After PSM, 964 patients with HR PCa and 538 patients with VHR PCa were included in each group. The 10-year OS and PCSS of men with HR PCa were $60.1 \%$ vs $40.9 \%$ and $90.6 \%$ vs $83.4 \%$, respectively. The 10 -year rate of OS and PCSS in men with VHR PCa were $55.9 \%$ vs $33.3 \%$ and $82.4 \%$ vs $75.6 \%$, respectively. The OS curve of patients with HR PCa revealed that RP was significantly better than EBRT in both overall cohort [HR: $0.533,95 \% \mathrm{Cl}(0.485 \sim 0.586), \mathrm{p}<0.001]$ and the matched cohort [HR: $0.703,95 \% \mathrm{Cl}(0.595 \sim 0.832)$, $\mathrm{p}<0.001$ ]. However, the PCSS curve of patients with HR PCa showed that RP was significantly better than EBRT in overall cohort [HR: $0.453,95 \% \mathrm{Cl}(0.368 \sim 0.559), \mathrm{p}<0.001$ ] but was similar to EBRT in matched cohort [HR: $0.820,95 \% \mathrm{Cl}(0.552 \sim 1.218), \mathrm{p}=0.327]$. As for patients with VHR PCa, RP was associated with better OS than EBRT whether in overall cohort [HR: $0.520,95 \% \mathrm{Cl}(0.457 \sim 0.592), \mathrm{p}<0.001]$ or matched cohort [0.695, $95 \% \mathrm{Cl}(0.551 \sim 0.876), p=0.002]$. The PCSS of RP was significantly better than that of EBRT in overall cohort [HR: $0.538,95 \% \mathrm{Cl}(0.422 \sim 0.685), \mathrm{p}<0.001]$, but was similar in matched cohort [HR: $0.787,95 \% \mathrm{Cl}(0.510$ $\sim 1.214), \mathrm{p}=0.281]$.
\end{abstract}

Conclusions: RP has more survival benefits than EBRT in men aged 75 years and older with HR or VHR PCa.

Key words: High-risk/very high-risk prostate cancer; radical prostatectomy; external beam radiation; survival; SEER

\section{Introduction}

Prostate cancer $(\mathrm{PCa})$ is the most common malignancy in the male genitourinary system, accounting for about $15 \%$ of all malignant tumors in the world. It ranks first in the incidence of male malignancies, and the second in male cancer-related deaths, seriously threatening the lives and health of male patients [1, 2]. In 2020, it is estimated that more than 191,930 men in the United States are newly diagnosed with PCa and 33,330 cases of PCa deaths [3]. With the widespread use of prostate-specific 
antigen (PSA), the incidence of PCa and the clinical stage of newly diagnosed PCa have changed dramatically. The proportion of early-stage PCa increased significantly, while the advanced PCa greatly reduced in newly diagnosed cases [4-6]. Among the newly diagnosed PCa patients, localized PCa accounts for approximately $80 \%[7,8]$. According to the guideline of National Comprehensive Cancer Network (NCCN) for prostate cancer, the high-risk (HR) PCa is defined as T3a, PSA $>20 \mathrm{ng} / \mathrm{ml}$, or Gleason Score (GS 8-10), and Very high-risk (VHR) as T3b-4, grade group 5, or $>4$ core positive grade group 4-5 [9]. $\mathrm{HR} / \mathrm{VHR} \mathrm{PCa}$ is characterized by recurrences and high cancer-related deaths. Besides, VHR PCa is more aggressive and associated with higher risks of cancer-specific mortality than HR PCa.

Nowadays, the main radical local treatments for $\mathrm{HR} / \mathrm{VHR}$ PCa are radical prostatectomy (RP) with/without antiandrogen treatment (ADT) and external beam radiation (EBRT). RP is the preferred choice of treatment for specific patients with localized diseases and life expectancy $>10$ years [10, 11]. However, its benefits vary in patients, especially in elderly men. In recent years, with the development of radiotherapy equipment and technology, the side effects of EBRT have remarkably reduced, making EBRT an effective and important treatment for HR/VHR PCa.

Even though many trials have evaluated the benefits of RP and EBRT in HR PCa and have shown that RP seems to have more survival benefits than EBRT. But it is still unclear whether RP remains superior to EBRT in elderly men (75 years and older) with HR PCa, or VHR PCa. The aim of this study was to evaluate the prognostic differences between RP (with or without ADT) and EBRT in elderly men (75 years and older) with localized HR PCa or VHR PCa.

\section{Materials and Methods}

\section{Data source}

The data of this study were derived from the Surveillance, Epidemiology and End Results (SEER) database with the software SEER* STAT. Elderly patients (aged 75 years and older) with localized PCa (cT1-4N0M0) diagnosed from January 1, 2004 to December 31, 2015 were retrospectively identified.

\section{Inclusion and exclusion criteria}

Patients were considered eligible if they met the following criteria: (1) patients with primary localized (cT1-4N0M0) PCa. (2) Patients were 75 years and older at the time of diagnosis. (3) Patients were diagnosed with HR PCa or VHR PCa according to the definition of NCCN guideline. (4) The treatments were RP (with or without ADT) or EBRT.
The following criteria were used for data exclusion: (1) Multiple tumors; (2) Important information such as PSA, GS, and TNM staging was incomplete or missing; (3) The survival status at the end of the follow-up was unclear, or the follow-up time was incomplete.

\section{Variables and main outcomes}

Patients' general information, tumor information, and survival status were collected. The variables included age $(75-79,80-84, \geq 85)$, race (white, black, other races including American Indian and Asian/Pacific Islander), marital status (married, unmarried, divorced or separated), T stage (T1, T2, T3, T4), PSA level ) $\leq 20 \mathrm{ng} / \mathrm{ml},>20 \mathrm{ng} / \mathrm{ml})$, GS $(<8,8-10)$, treatment (RP, EBRT), survival time, living status (alive or dead) and cancer-specific living status (alive, died for other reasons, died for prostate cancer). The main outcomes of this study were overall survival (OS) and prostate cancer-specific survival (PCSS) in overall cohort and matched cohort.

\section{Statistical analyses}

Chi-square test was used to assess the baseline characteristics between the RP group and EBRT group. Propensity-score matching (PSM) based on the nearest-neighbor matching principle was conducted to balance the covariates in two groups. OS and PCSS curves were constructed with Kaplan-Meier (K-M) analysis, and 5-year and 10-year OS and PCSS were calculated by survival tables. Univariate COX analysis was used for each variable to obtain the relevant parameters of OS and PCSS prognosis. Multivariate Cox analysis was performed on the variable with $\mathrm{P}<0.1$. The effects of various factors on the survival and prognosis were evaluated by hazard ratio (HR) values with $95 \%$ confidence interval $(95 \% \mathrm{CI})$. The above statistical operations were performed with the software of SPSS 25 and Graph prism 7. P $<0.05$ was considered statistically significant.

\section{Results}

\section{Patient characteristics}

11698 patients with HR PCa were identified, with a median age of 76 (78-81) years with 1307 patients in RP group and 10391 patients in EBRT group. The median follow-up time is 60 (32-90) months. After PSM, 964 patients were left in each group. The baseline characteristics of two groups in both overall cohort and matched cohort were shown in Table 1.

4415 patients with VHR PCa were identified, with 742 patients in RP group and 3673 patients in EBRT group. 538 patients remained in each group 
after PSM. The baseline characteristics of overall cohort and matched cohort were presented in Table 2.

\section{Survival outcomes}

\section{5-year and 10-year OS and PCSS rates}

The 10-year OS rate of patients with HR PCa in RP group and EBRT group were $60.1 \%$ vs $40.9 \%$ $(\mathrm{P}<0.001)$ in overall cohort, and $56.3 \%$ vs $45 \%$ $(\mathrm{P}<0.001)$ in matched cohort. The 5-year and 10-year PCSS rate of the RP group and EBRT group were $90.6 \%$ vs $83.4 \%(\mathrm{P}<0.001)$ in overall cohort, and $88.0 \%$ vs $87.0 \%(\mathrm{P}=0.136)$ in matched cohort. The 5 - and 10 year OS and PCSS rates in two cohorts were presented in Table 3.

The 10-year OS rate of patients with VHR PCa in RP group and EBRT group were $55.9 \%$ vs $33.3 \%$ $(\mathrm{P}<0.001)$ in overall cohort, and $56.9 \%$ vs $41.5 \%$ $(\mathrm{P}<0.001)$ in matched cohort. The 10-year PCSS rate of the RP group and EBRT group was $82.4 \%$ vs $75.6 \%$ $(\mathrm{P}<0.001)$ in overall cohort, and $82.9 \%$ vs $79.2 \%$ $(\mathrm{P}=0.073)$ in matched cohort. These results were also revealed in Table 3.
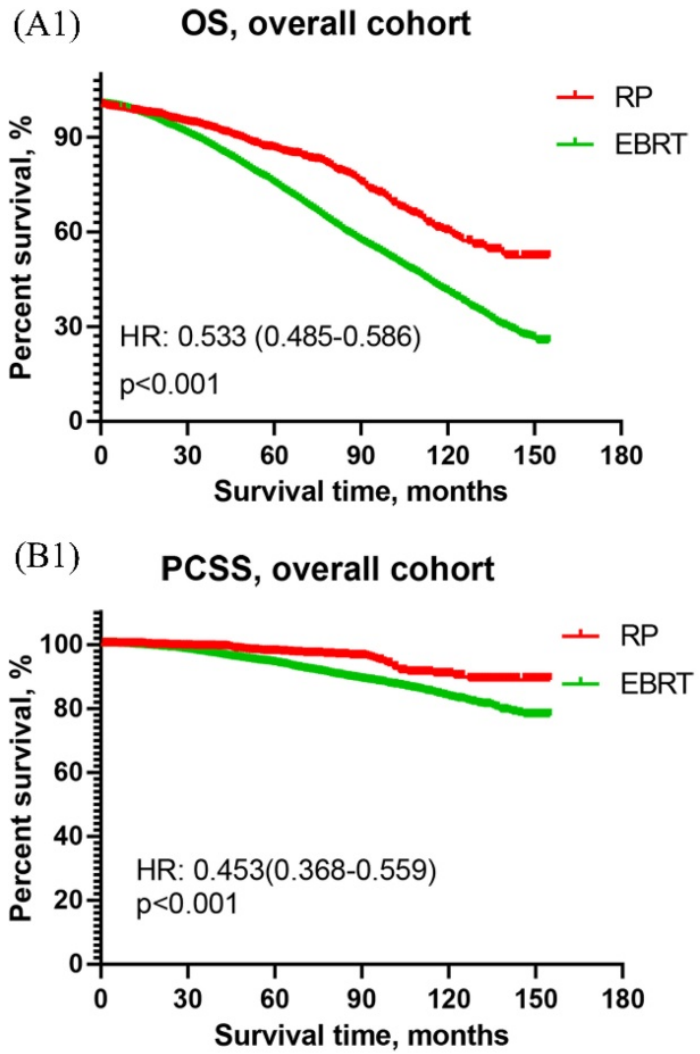

\section{Survival curves}

Survival curves of men with HR PCa

The OS curves of men with HR PCa showed that $\mathrm{RP}$ was associated with significantly better OS than EBRT in both overall cohort [HR: 0.533, 95\% CI (0.485 0.586), $\mathrm{p}<0.001$ ] and matched cohort [HR: $0.703,95 \% \mathrm{CI}(0.595 \sim 0.832), \mathrm{p}<0.001]$. The results were presented in Figure 1A. As for the results of PCSS curves, RP was obviously better than EBRT in overall cohort [HR: $0.453,95 \%$ CI (0.368 0.559), p<0.001] but they two were similar in matched cohort [HR: 0.820, $95 \%$ CI (0.552 1.218), $p=0.327]$. The results were shown in Figure 1B.

\section{Survival curves of men with VHR PCa}

As shown in Figure 2A, the OS curve of men with VHR PCa revealed that RP was apparently better than EBRT in both overall cohort [HR:0.520, 95\%CI (0.457 0.592), $\mathrm{p}<0.001$ ] and matched cohort [HR: $0.695,95 \%$ CI $(0.551 \sim 0.876), p=0.002]$. The PCSS curve of men with VHR PCa showed that RP had significantly better PCSS than EBRT in overall cohort [HR: $0.538,95 \%$ CI $(0.422 \sim 0.685), \mathrm{p}<0.001$ ], and had similar results with EBRT in matched cohort [HR: $0.787,95 \%$ CI $(0.510 \sim 1.214), \mathrm{p}=0.281]$. This was presented in Figure 2B.

\section{(A2) OS, matched cohort}

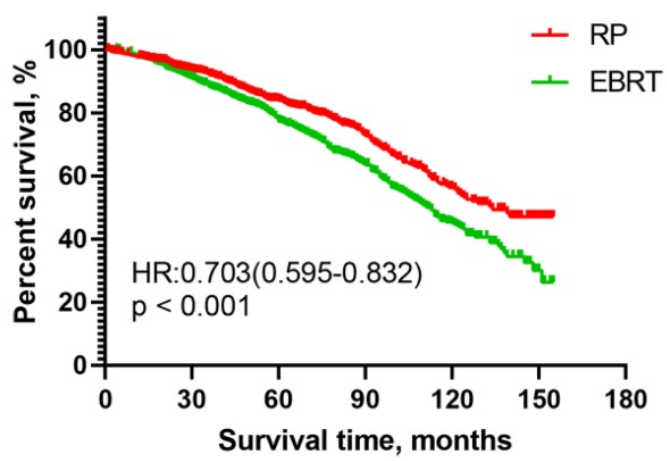

(B2)

\section{PCSS, matched cohort}

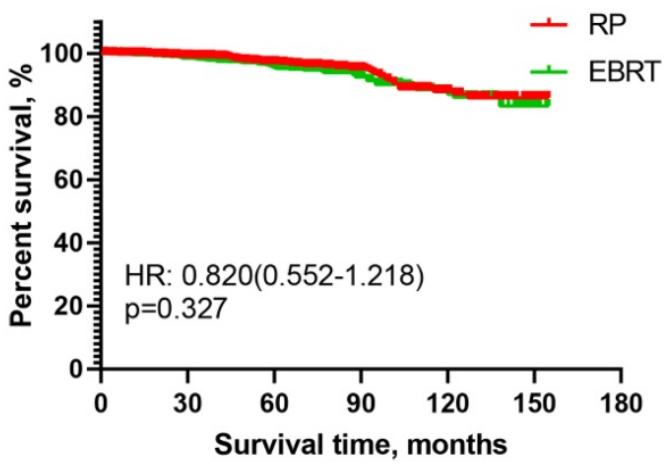

Figure 1. Overall survival and prostate cancer-specific survival curves of RP and EBRT groups for aging 75 and older patients with high-risk prostate cancer. (A1) Overall survival curve of RP and EBRT in overall cohort. (A2) Overall survival curve in propensity matched cohort. (B1) Prostate cancer-specific survival curve of RP and EBRT in overall cohort. (B2) Prostate cancer-specific survival curve of RP and EBRT in propensity matched cohort. 
Table 1. The baseline characteristics of patients with high-risk prostate cancer

\begin{tabular}{|c|c|c|c|c|c|c|}
\hline \multirow[t]{2}{*}{ Variables } & \multicolumn{3}{|l|}{ Overall cohort } & \multicolumn{3}{|c|}{ Propensity-matched cohort } \\
\hline & $\mathrm{RP}(\mathrm{n}=1307)$ & EBRT $(n=10391)$ & $\mathrm{P}$ & $\mathrm{RP}(\mathrm{n}=964)$ & EBRT $(n=964)$ & $\mathrm{P}$ \\
\hline \multicolumn{7}{|l|}{ Age (years) } \\
\hline Median (IQR) & $76(75-78)$ & $78(76-81)$ & & $77(76-78)$ & $79(78-79)$ & \\
\hline \multicolumn{7}{|l|}{ Age, $n(\%)$} \\
\hline $75-79$ & $1143(87.5)$ & $6551(63)$ & $<0.001$ & $806(83.6)$ & $796(82.6)$ & 0.837 \\
\hline $80-84$ & $137(10.5)$ & $3131(30.1)$ & & 132 (13.7) & $140(14.5)$ & \\
\hline$\geq 85$ & $27(2.1)$ & $709(6.8)$ & & $26(2.7)$ & $28(2.9)$ & \\
\hline \multicolumn{7}{|l|}{ Race, n (\%) } \\
\hline White & $1115(85.3)$ & $8474(81.6)$ & $<0.001$ & $832(86.3)$ & $820(85.1)$ & 0.837 \\
\hline Black & $66(5)$ & $939(9)$ & & $49(5.1)$ & $51(5.3)$ & \\
\hline Others & $116(8.9)$ & $864(8.3)$ & & $78(8.1)$ & $86(8.9)$ & \\
\hline Unclear & $10(0.8)$ & $114(1.1)$ & & $5(0.5)$ & $7(0.7)$ & \\
\hline \multicolumn{7}{|c|}{ Marital status, $\mathrm{n}(\%)$} \\
\hline Married & 1014 (77.6) & $7229(69.6)$ & $<0.001$ & $717(74.4)$ & $718(74.5)$ & 0.930 \\
\hline Unmarried & $76(5.8)$ & $634(6.1)$ & & $63(6.5)$ & $64(6.6)$ & \\
\hline Separated & $163(12.5)$ & $1681(16.2)$ & & $138(14.3)$ & $131(13.6)$ & \\
\hline Unclear & $54(4.1)$ & $847(8.2)$ & & $46(4.8)$ & $51(5.3)$ & \\
\hline \multicolumn{7}{|l|}{ T stage, $\mathrm{n}(\%)$} \\
\hline $\mathrm{T} 1-2$ & $550(42.1)$ & $9662(93)$ & $<0.001$ & $550(57.1)$ & $544(56.4)$ & 0.783 \\
\hline T3a & 757 (57.9) & $729(7)$ & & $414(42.9)$ & $420(43.6)$ & \\
\hline \multicolumn{7}{|l|}{ GS, n (\%) } \\
\hline$<8$ & $545(41.7)$ & $1994(19.2)$ & $<0.001$ & $282(29.3)$ & $276(28.6)$ & 0.763 \\
\hline $8-10$ & $762(58.3)$ & $8397(80.8)$ & & $682(70.7)$ & $688(71.4)$ & \\
\hline \multicolumn{7}{|l|}{ PSA (ng/ml) } \\
\hline Median (IQR) & $7.8(5.4-12.2)$ & $11.8(7.1-23.5)$ & & $8.0(5.5-14.3)$ & $9.5(6.3-16.1)$ & \\
\hline \multicolumn{7}{|c|}{ PSA (ng/ml), n (\%) } \\
\hline$<20$ & $1131(86.5)$ & $6998(67.3)$ & $<0.001$ & $793(82.3)$ & $799(82.9)$ & 0.719 \\
\hline$>20$ & $176(13.5)$ & $3393(32.7)$ & & $171(17.7)$ & $165(17.1)$ & \\
\hline \multicolumn{7}{|c|}{ Follow-up time (months) } \\
\hline Median (IQR) & $62(33-93)$ & $60(32-89)$ & & $58.5(32-89.8)$ & $62(33-91)$ & \\
\hline
\end{tabular}

IQR, interquartile range; RP, radical prostatectomy; EBRT, external beam radiotherapy; GS: Gleason score; PSA, prostate-specific antigen.

(A1) OS, overall cohort

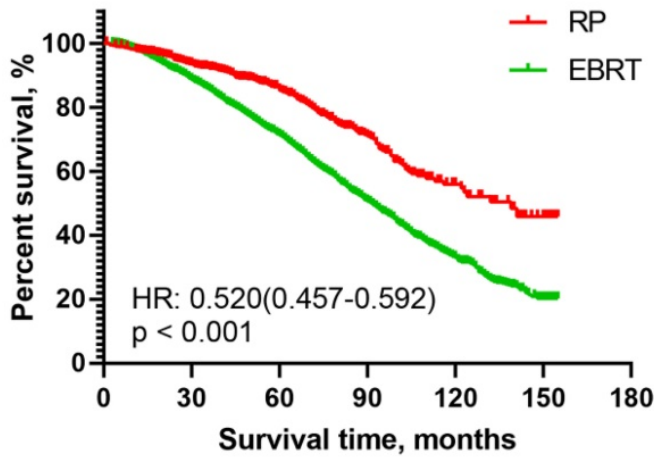

(B1)

PCSS, overall cohort

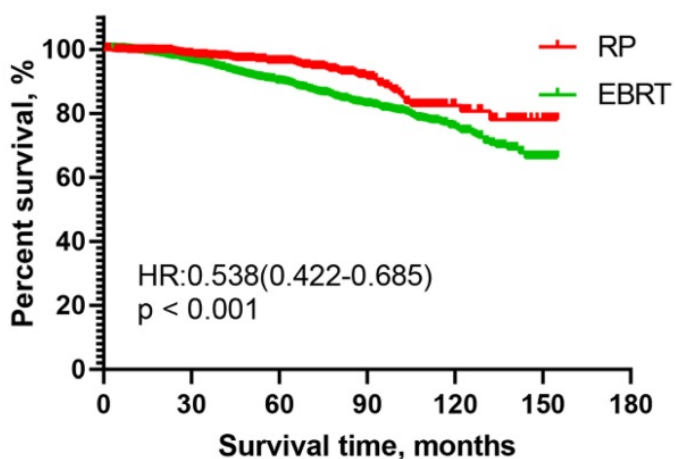

(A2)

OS, matched cohort

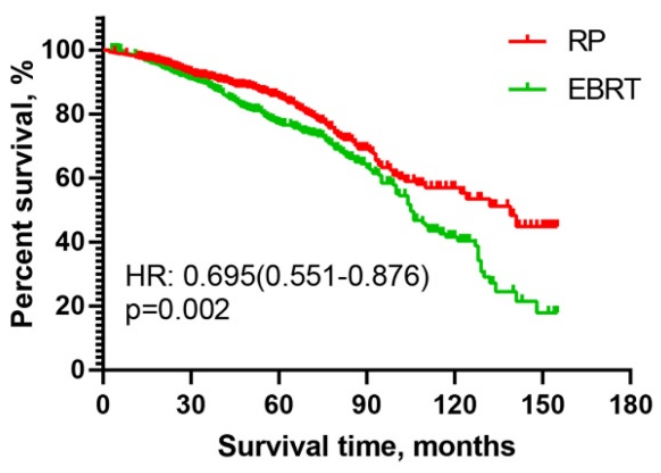

PCSS, matched cohort

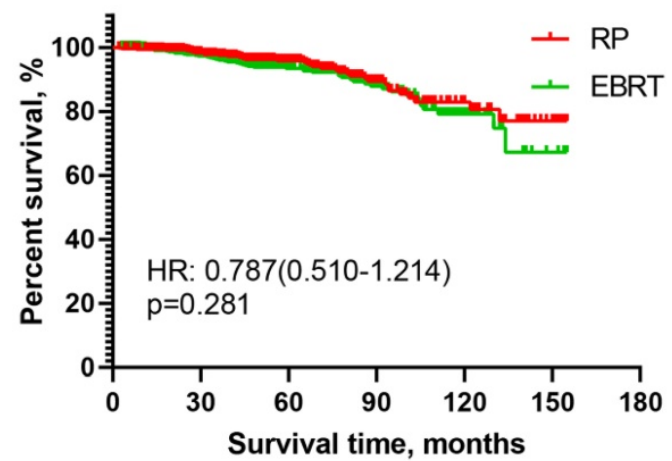

Figure 2. Overall survival and prostate cancer-specific survival curves of RP and EBRT groups for aging 75 and older patients with very high-risk prostate cancer. (A1) Overall survival curve of RP and EBRT in overall cohort. (A2) Overall survival curve in propensity matched cohort. (B1) Prostate cancer-specific survival curve of RP and EBRT in overall cohort. (B2) Prostate cancer-specific survival curve of RP and EBRT in propensity matched cohort. 
Table 2. The baseline characteristics of patients with very high-risk prostate cancer

\begin{tabular}{|c|c|c|c|c|c|c|}
\hline \multirow[t]{2}{*}{ Variables } & \multicolumn{3}{|l|}{ Overall cohort } & \multicolumn{3}{|c|}{ Propensity-matched cohort } \\
\hline & $\mathrm{RP}(\mathrm{n}=742)$ & EBRT $(n=3673)$ & $\mathrm{P}$ & $\mathrm{RP}(\mathrm{n}=538)$ & EBRT $(n=538)$ & $\mathrm{P}$ \\
\hline \multicolumn{7}{|l|}{ Age (years) } \\
\hline Median (IQR) & $76(75-78)$ & $78(76-82)$ & & $76(75-78)$ & $76(75-79)$ & \\
\hline \multicolumn{7}{|l|}{ Age, $n(\%)$} \\
\hline $75-79$ & $638(86)$ & $2222(60.5)$ & $<0.001$ & $440(81.8)$ & $437(81.2)$ & 0.961 \\
\hline $80-84$ & $94(12.7)$ & $1163(31.7)$ & & $88(16.4)$ & $90(16.7)$ & \\
\hline$\geq 85$ & $10(1.3)$ & $288(7.8)$ & & $10(1.9)$ & $11(2)$ & \\
\hline \multicolumn{7}{|l|}{ Race, n (\%) } \\
\hline White & $639(86.1)$ & 3070 (83.6) & $<0.001$ & 477 (88.7) & $466(86.6)$ & 0.593 \\
\hline Black & $38(5.1)$ & $279(7.6)$ & & $22(4.1)$ & $28(5.2)$ & \\
\hline Others & $60(8.1)$ & $287(7.8)$ & & $36(6.7)$ & $38(7.1)$ & \\
\hline Unclear & $5(0.7)$ & $37(1)$ & & $3(0.6)$ & $6(1.1)$ & \\
\hline \multicolumn{7}{|c|}{ Marital status, n (\%) } \\
\hline Married & $586(79)$ & $2587(70.4)$ & $<0.001$ & 417 (77.5) & 409 (76) & 0.867 \\
\hline Unmarried & $37(5)$ & $218(5.9)$ & & $26(4.8)$ & $31(5.8)$ & \\
\hline Separated & $91(12.3)$ & $617(16.8)$ & & 75 (13.9) & $75(13.9)$ & \\
\hline Unclear & $28(3.8)$ & $251(6.8)$ & & $20(3.7)$ & $23(4.3)$ & \\
\hline T stage, $\mathrm{n}(\%)$ & & & & & & 0.894 \\
\hline $\mathrm{T} 1-2$ & $159(21.4)$ & $2989(81.4)$ & $<0.001$ & $159(29.6)$ & $161(29.9)$ & \\
\hline T3-4 & $583(78.6)$ & $684(18.6)$ & & $379(70.4)$ & $377(70.1)$ & \\
\hline \multicolumn{7}{|l|}{ GS, n (\%) } \\
\hline$<8$ & $205(27.6)$ & $128(3.5)$ & $<0.001$ & $104(19.3)$ & $105(19.5)$ & 0.939 \\
\hline $8-10$ & $537(72.4)$ & $3545(96.5)$ & & $434(80.7)$ & $433(80.5)$ & \\
\hline \multicolumn{7}{|l|}{ PSA (ng/ml) } \\
\hline Median (IQR) & $8.2(5.7-12.6)$ & $10.7(6.8-19.3)$ & & $8.4(5.8-13.7)$ & $9.0(6.2-14.3)$ & \\
\hline \multicolumn{7}{|c|}{ PSA (ng/ml), n (\%) } \\
\hline$<20$ & $671(90.4)$ & $2804(76.3)$ & $<0.001$ & $473(87.9)$ & $483(89.8)$ & 0.333 \\
\hline$>20$ & $71(9.6)$ & $869(23.7)$ & & $65(12.1)$ & $55(10.2)$ & \\
\hline \multicolumn{7}{|c|}{ Follow-up time (months) } \\
\hline Median (IQR) & $54(27-85)$ & $53(29-82)$ & & $53(26-81)$ & $56(31-83)$ & \\
\hline
\end{tabular}

Table 3. 5-year and 10-year OS and PCSS for men aging 75 and older with high-risk and very high-risk prostate cancer

\begin{tabular}{|c|c|c|c|c|c|c|c|}
\hline \multirow[t]{2}{*}{ Variables } & & \multicolumn{3}{|l|}{ Overall cohort } & \multicolumn{3}{|c|}{ Propensity-matched cohort } \\
\hline & & $\mathrm{RP}(\%)$ & EBRT (\%) & $\mathrm{P}$ & $\mathrm{RP}(\%)$ & EBRT (\%) & $\mathrm{P}$ \\
\hline \multicolumn{8}{|c|}{ High risk $P C a$} \\
\hline \multirow[t]{2}{*}{ OS } & 5 years & $86.2(84 \% \sim 88.4 \%)$ & $75.2(74.2 \% \sim 76.2 \%)$ & $<0.001$ & $84.1(81.4 \% \sim 86.8 \%)$ & $77.3(74.4 \% \sim 80.2 \%)$ & $<0.001$ \\
\hline & 10 years & $60.1(55.6 \% \sim 64.6 \%)$ & $40.9(39.5 \% \sim 42.3 \%)$ & $<0.001$ & $56.3(50.8 \% \sim 61.8 \%)$ & $45(40.1 \% \sim 49.9 \%)$ & $<0.001$ \\
\hline \multirow[t]{2}{*}{ PCSS } & 5 years & $97.5(96.5 \% \sim 98.5 \%)$ & $94(93.4 \% \sim 94.6 \%)$ & 0.054 & $97(95.6 \% \sim 98.4 \%)$ & $95.4(93.8 \% \sim 97 \%)$ & 0.068 \\
\hline & 10 years & $90.6(87.5 \% \sim 93.7 \%)$ & $83.4(82 \% \sim 84.8 \%)$ & $<0.001$ & $88(83.9 \% \sim 92.1 \%)$ & $87(83.1 \% \sim 90.9 \%)$ & 0.136 \\
\hline \multicolumn{8}{|c|}{ Very high risk $\mathrm{PCa}$} \\
\hline \multirow[t]{2}{*}{ OS } & 5 years & $85.3(82.4 \% \sim 88.2 \%)$ & $71.3(69.5 \% \sim 73.1 \%)$ & $<0.001$ & $85.1(81.6 \% \sim 88.6 \%)$ & $76.9(72.8 \% \sim 81 \%)$ & $<0.001$ \\
\hline & 10 years & $55.9(49.4 \% \sim 62.4 \%)$ & $33.3(30.8 \% \sim 35.8 \%)$ & $<0.001$ & $56.9(49.5 \% \sim 64.3 \%)$ & $41.5(34.2 \% \sim 48.8 \%)$ & $<0.001$ \\
\hline \multirow[t]{2}{*}{ PCSS } & 5 years & $96.1(94.3 \% \sim 97.9 \%)$ & $89.8(88.6 \% \sim 91 \%)$ & $<0.001$ & $95.9(93.9 \% \sim 97.9 \%)$ & $93.4(91 \% \sim 95.8 \%)$ & 0.131 \\
\hline & 10 years & $82.4(76.7 \% \sim 88.1 \%)$ & $75.6(72.9 \% \sim 78.3 \%)$ & $<0.001$ & $82.9(76.4 \% \sim 89.4 \%)$ & $79.2(72.3 \% \sim 86.1 \%)$ & 0.073 \\
\hline
\end{tabular}

OS: overall survival; PCSS: prostate cancer-specific survival; RP: radical prostatectomy; EBRT: external beam radiotherapy.

\section{Multivariate COX analysis for OS and PCSS}

Multivariate COX analysis results of the OS and PCSS were presented in Table 4. Factors related to the risk of death included age, race, marital status, T-stage, Gleason score, PSA, and treatments. With RP as the reference, The HR and $95 \% \mathrm{CI}$ of EBRT for overall mortality of men with HR and VHR PCa were 1.62 (1.42 1.86), and 1.55 (1.29 1.87), respectively. The HR and $95 \% \mathrm{CI}$ of EBRT for cancer-specific mortality of men with HR and VHR PCa were 1.86 (1.35 2.55), and 1.58 (1.12 2.21), respectively.

\section{Discussion}

Age is an important factor that has an impact on the prognosis of HR/VHR PCa and affects the choice of treatments for clinicians and patients. The older patients at diagnosis are more likely to be at high risk and less likely to receive curative local therapies [12]. RP (with/without ADT) and EBRT are two main curative local therapies for non-metastatic HR/VHR PCa. Even though many studies have evaluated the survival difference of RP and EBRT and have revealed that RP seems to have more survival benefits than EBRT in men with HR or VHR PCa. However, the benefits seem to be inconclusive for elderly men, especially those aged 75 and older because their life expectancy varies.

A total of 11698 patients aged 75 and older with localized HR PCa and 4415 with VHR PCa were included in our study. After PSM, 1928 patients with HR PCa and 1076 patients with VHR PCa were left. 
The 10 years OS rate of men with HR PCa in RP and EBRT group were $60.1 \%$ vs. $40.9 \%$ in overall cohort and $56.3 \%$ vs. $45.0 \%$ in matched cohort. The 10 -year PCSS rate of those in RP and EBRT groups were $90.6 \%$ vs. $83.4 \%$ in overall cohort and $88.0 \%$ vs. $87.0 \%$ in matched cohort, respectively. This result was consistent with many studies' findings [13-15]. Boorjian et al. [16] analyzed 1,238 patients with localized high-risk PCa who underwent RP and 609 patients receiving EBRT. The10-year PCSS rate was $92 \%$ and $88 \%$, respectively $(p=0.06)$. Eifler et al. [17] showed that men who underwent RP had a 10-year PCSS rate of more than $90 \%$. A study [18] analyzed the prognosis of 234 patients over 80 years old who underwent RP surgery. In this study, the 10-year OS rate was $51 \%$ and 10-year PCSS was 9.9\%, similar to our results. In addition, Bandini et al. [19] analyzed the survival of old patients (>75 years) with T1-2 localized PCa who received local treatments (RP or EBRT). Its results showed that the 10-year prostate cancer-specific mortality in RP and EBRT groups were $4.3 \%$ and $6.1 \%$, respectively.

The VHR PCa is more aggressive and is associated with a poorer prognosis for the patients than HR PCa. Pompe et al. [20] found that VHR PCa had obviously worse biochemical recurrence-free survival, metastatic progression-free survival, OS and PCSS rates compared to HR PCa. In our study, the 10-year OS rate of RP and EBRT group were 55.9\% vs.33.3\% in overall cohort and $56.9 \%$ vs $41.5 \%$ in matched cohort. The 10-year PCSS rate of two groups were $82.4 \%$ vs $75.6 \%$ in overall cohort and $82.9 \%$ vs $79.2 \%$ in matched cohort. As we know, there were few studies evaluating the prognosis differences between RP and EBRT in aging 75 and older patients with VHR PCa. Sundi et al.[21] analyzed the outcomes of HR PCa and VHR PCa after the treatment of RP, and found that the 5- year all-cause mortality and cancer-specific mortality were 0.033 and 0.007 in patients with HR PCa and 0.006 and 0.045 in those with VHR PCa. Reichard et al. [22] reported that the all-cause mortality rate of $\mathrm{RP}$ and radiotherapy for patients with HR PCa and VHR PCa were $9.5 \%$ and $8.2 \%$.

The results of K-M analysis showed that men with HR PCa or VHR PCa in RP group were associated with obviously better OS than those in EBRT group no matter in overall cohort or matched cohort. This result revealed that RP had more OS benefits than EBRT in aging 75 and older patients with HR PCa and even with VHR PCa. As for the PCSS results in overall cohort, RP group was significantly better than EBRT group in men with HR PCa or VHR PCa. However, the difference between two groups was not significant in matched cohort. Therefore, we could find out that RP had more survival benefits than EBRT in aging 75 and older men with HR PCa and with VHR PCa.

Table 4. Multivariate COX analysis for high-risk and very high-risk patients in matched groups

\begin{tabular}{|c|c|c|c|c|c|c|c|c|}
\hline Risk factors & High-risk & & & & Very high-risk & & & \\
\hline HR $(95 \%$ CI $)$ & OS & & PCSS & & OS & & PCSS & \\
\hline Age & & & & & & & & \\
\hline $75-79$ & 1 & Ref. & 1 & Ref. & 1 & Ref. & 1 & Ref. \\
\hline $80-84$ & $1.44(1.35 \sim 1.54)$ & $<0.001$ & $1.36(1.17 \sim 1.57)$ & $<0.001$ & $1.4(1.26 \sim 1.56)$ & $<0.001$ & $1.21(0.98 \sim 1.48)$ & 0.073 \\
\hline$\geq 85$ & $2.08(1.86 \sim 2.32)$ & $<0.001$ & $1.79(1.4 \sim 2.28)$ & $<0.001$ & $1.85(1.56 \sim 2.2)$ & $<0.001$ & $1.37(0.97 \sim 1.93)$ & 0.072 \\
\hline Race & & & & & & & & \\
\hline White & 1 & Ref. & 1 & Ref. & 1 & Ref. & 1 & Ref. \\
\hline Black & $1.13(1.01 \sim 1.26)$ & $<0.001$ & $1.26(1.00 \sim 1.59)$ & 0.049 & $1.25(1.04 \sim 1.5)$ & 0.019 & $1.35(0.97 \sim 1.88)$ & 0.076 \\
\hline Others & $0.79(0.71 \sim 0.89)$ & $<0.001$ & $0.89(0.69 \sim 1.14)$ & 0.360 & $0.78(0.64 \sim 0.95)$ & 0.012 & $0.71(0.49 \sim 1.03)$ & 0.072 \\
\hline Marital statu & & & & & & & & \\
\hline Married & 1 & Ref. & 1 & Ref. & 1 & Ref. & 1 & Ref. \\
\hline Unmarried & $1.14(1.05 \sim 1.24)$ & 0.029 & $1.05(0.79 \sim 1.40)$ & 0.744 & $1.06(0.86 \sim 1.32)$ & 0.579 & $0.89(0.59 \sim 1.34)$ & 0.576 \\
\hline Divorced & $1.34(1.26 \sim 1.42)$ & $<0.001$ & $1.19(1.00 \sim 1.42)$ & 0.048 & 1.31 (1.16 1.48) & $<0.001$ & $0.95(0.74 \sim 1.22)$ & 0.685 \\
\hline T stage & & & & & & & & \\
\hline $\mathrm{T} 1$ & 1 & Ref. & 1 & Ref. & 1 & Ref. & 1 & Ref. \\
\hline $\mathrm{T} 2$ & $1.16(1.09 \sim 1.24)$ & $<0.001$ & $1.25(1.08 \sim 1.44)$ & 0.003 & $1.14(1.02 \sim 1.28)$ & 0.021 & $1.2(0.97 \sim 1.5)$ & 0.094 \\
\hline $\mathrm{T} 3$ & $1.2(1.060 \sim 1.34)$ & $<0.001$ & $1.58(1.24 \sim 2.01)$ & $<0.001$ & $1.0(0.84 \sim 1.18)$ & 0.974 & $1.4(1.04 \sim 1.87)$ & 0.025 \\
\hline $\mathrm{T} 4$ & & & & & $1.59(1.22 \sim 2.08)$ & 0.001 & $3.25(2.09 \sim 5.07)$ & $<0.001$ \\
\hline PSA & & & & & & & & \\
\hline$<20$ & 1 & Ref. & 1 & Ref. & 1 & Ref. & 1 & Ref. \\
\hline$>20$ & $1.26(1.16 \sim 1.36)$ & $<0.001$ & 1.75 (1.49 2.05) & $<0.001$ & $1.28(1.14 \sim 1.44)$ & $<0.001$ & $1.84(1.5 \sim 2.25)$ & $<0.001$ \\
\hline Gleason sco & & & & & & & & \\
\hline$<8$ & 1 & Ref. & 1 & Ref. & 1 & Ref. & 1 & Ref. \\
\hline 8 & $1.21(1.10 \sim 1.33)$ & $<0.001$ & $1.82(1.45 \sim 2.28)$ & $<0.001$ & $1.06(0.79 \sim 1.43)$ & 0.697 & $0.94(0.5 \sim 1.79)$ & 0.855 \\
\hline $9-10$ & $1.52(1.38 \sim 1.68)$ & $<0.001$ & $3.52(2.82 \sim 4.39)$ & $<0.001$ & $1.18(0.93 \sim 1.5)$ & 0.167 & $2.31(1.44 \sim 3.72)$ & 0.001 \\
\hline Treatment & & & & & & & & \\
\hline $\mathrm{RP}$ & 1 & Ref. & 1 & Ref. & 1 & Ref. & 1 & Ref. \\
\hline EBRT & $1.62(1.42 \sim 1.86)$ & $<0.001$ & $1.86(1.35 \sim 2.55)$ & $<0.001$ & 1.55 (1.29 1.87) & $<0.001$ & $1.58(1.12 \sim 2.21)$ & 0.009 \\
\hline
\end{tabular}

OS: overall survival; PCSS: prostate cancer-specific survival; HR: hazard ratio; Ref: reference; PSA: prostate-specific antigen; RP: radical prostatectomy; EBRT: external beam radiotherapy. 
The COX analysis showed that risk factors associated with prognosis in patients with localized high-risk prostate cancer were age, race, marital status, T stage, PSA, Gleason score, and treatment. A similar study [23] found that risk factors for PCa include family history, genetics, age, ethnicity, and tumor characteristics. According to the results of COX analysis, the risk of death in the EBRT group was significantly higher than that in the RP group, which means that the prognosis of EBRP is worse than that of RP. It was consistent with the results of the OS and PCSS curves. Two meta-analyses reported that OS and PCSS of RP were significantly better than those of EBRT in high-risk PCa patients [24, 25]. RP seemed to be more likely to reduce the long-term total mortality and prostate cancer-specific mortality in elderly patients with high-risk prostate cancer.

Even though our study analyzed a large sample of patients, there were still some limitations in our study. The limitations of our study were as follows: (1) Our study was still a retrospective analysis in which there were some unavoidable confounders and risk biases. This may have an impact and interference with the results. (2) Even though we analyzed men aged 75 years and older in our study, the large percentages were in 75-79 years old subgroup and only a few percentages of patients were in $\geq 85$ years subgroup. Therefore, our results might not be suitable for all patients aging older than 75 years. (3) Besides the survival benefits, many factors of individuals such as health status (associated with chronic diseases or not, food intake, body mass index, mobility, etc.), patients' willingness, life expectancy, the quality of life after treatments should be fully considered when choosing RP as the main treatment. RP should be performed in the selected patients but not all elderly patients aging 75 and over. (4) Our study only focused on the analysis of survival prognosis, many important clinical outcomes were not analyzed, such as post-treatment comorbidities, quality of life of patients. It is not appropriate to evaluate two treatments only by survival prognosis.

\section{Conclusion}

Among men aged 75 and older with HR PCa and even VHR PCa, RP was associated with more survival benefits than EBRT. However, with the limitations of our study, high-quality studies are needed for future evaluations.

\section{Acknowledgements}

All data were from the public database; no ethical approval was required.

\section{Competing Interests}

The authors have declared that no competing interest exists.

\section{References}

1. Ferlay J, Soerjomataram I, Dikshit R, Eser S, Mathers C, Rebelo M, et al. Cancer incidence and mortality worldwide: sources, methods and major patterns in GLOBOCAN 2012. International journal of cancer. 2015; 136: E359-86.

2. Torre LA, Siegel RL, Ward EM, Jemal A. Global Cancer Incidence and Mortality Rates and Trends--An Update. Cancer epidemiology, biomarkers \& prevention : a publication of the American Association for Cancer Research, cosponsored by the American Society of Preventive Oncology. 2016; 25: 16-27.

3. Siegel RL, Miller KD, Jemal A. Cancer statistics, 2020. CA: a cancer journal for clinicians. 2020; 70: 7-30.

4. Loeb S, Bjurlin MA, Nicholson J, Tammela TL, Penson DF, Carter HB, et al. Overdiagnosis and overtreatment of prostate cancer. European urology. 2014; 65: 1046-55

5. Collin SM, Martin RM, Metcalfe C, Gunnell D, Albertsen PC, Neal D, et al, Prostate-cancer mortality in the USA and UK in 1975-2004: an ecological study. The Lancet Oncology. 2008; 9: 445-52.

6. Makarov DV, Trock BJ, Humphreys EB, Mangold LA, Walsh PC, Epstein JI, et al. Updated nomogram to predict pathologic stage of prostate cancer given prostate-specific antigen level, clinical stage, and biopsy Gleason score (Partin tables) based on cases from 2000 to 2005. Urology. 2007; 69: 1095-101.

7. Newcomer LM, Stanford JL, Blumenstein BA, Brawer MK. Temporal trends in rates of prostate cancer: declining incidence of advanced stage disease, 1974 to 1994. The Journal of urology. 1997; 158: 1427-30.

8. Winter A, Sirri E, Jansen L, Wawroschek F, Kieschke J, Castro FA, et al. Comparison of prostate cancer survival in Germany and the USA: can differences be attributed to differences in stage distributions? BJU international. 2017; 119: 550-9.

9. Mohler JL, Antonarakis ES, Armstrong AJ, D'Amico AV, Davis BJ, Dorff T, et al. Prostate Cancer, Version 2.2019, NCCN Clinical Practice Guidelines in Oncology. Journal of the National Comprehensive Cancer Network : JNCCN. 2019; 17: 479-505.

10. Mottet N, Bellmunt J, Bolla M, Briers E, Cumberbatch MG, De Santis M, et al. EAU-ESTRO-SIOG guidelines on prostate cancer. Part 1: screening, diagnosis, and local treatment with curative intent. European urology. 2017; 71: 618-29.

11. Heidenreich A, Bastian PJ, Bellmunt J, Bolla M, Joniau S, van der Kwast T, et al. EAU guidelines on prostate cancer. part 1: screening, diagnosis, and local treatment with curative intent-update 2013. European urology. 2014; 65: 124-37.

12. Bechis SK, Carroll PR, Cooperberg MR. Impact of age at diagnosis on prostate cancer treatment and survival. Journal of clinical oncology : official journal of the American Society of Clinical Oncology. 2011; 29: 235-41.

13. Abdollah F, Sun M, Thuret R, Jeldres C, Tian Z, Briganti A, et al. A competing-risks analysis of survival after alternative treatment modalities for prostate cancer patients: 1988-2006. European urology. 2011; 59: 88-95.

14. Sooriakumaran P, Nyberg T, Akre O, Haendler L, Heus I, Olsson M, et al. Comparative effectiveness of radical prostatectomy and radiotherapy in prostate cancer: observational study of mortality outcomes. Bmj. 2014; 348: g1502.

15. Hoffman RM, Koyama T, Fan KH, Albertsen PC, Barry MJ, Goodman M, et al. Mortality after radical prostatectomy or external beam radiotherapy for localized prostate cancer. Journal of the National Cancer Institute. 2013; 105: 711-8.

16. Booriian SA, Karnes RJ, Viterbo R, Rangel LJ, Bergstralh EJ, Horwitz EM, et al. Long-term survival after radical prostatectomy versus external-beam radiotherapy for patients with high-risk prostate cancer. Cancer. 2011; 117: 2883-91.

17. Eifler JB, Humphreys EB, Agro M, Partin AW, Trock BJ, Han M. Causes of death after radical prostatectomy at a large tertiary center. The Journal of urology. 2012; 188: 798-801.

18. Dell'Oglio P, Zaffuto E, Boehm K, Trudeau V, Larcher A, Tian Z, et al. Long-term survival of patients aged 80 years or older treated with radical prostatectomy for prostate cancer. European journal of surgical oncology : the journal of the European Society of Surgical Oncology and the British Association of Surgical Oncology. 2017; 43: 1581-8.

19. Bandini M, Pompe RS, Marchioni M, Tian Z, Gandaglia G, Fossati N, et al. Radical prostatectomy or radiotherapy reduce prostate cancer mortality in elderly patients: a population-based propensity score adjusted analysis. World journal of urology. 2018; 36: 7-13.

20. Pompe RS, Karakiewicz PI, Tian Z, Mandel P, Steuber T, Schlomm T, et al. Oncologic and Functional Outcomes after Radical Prostatectomy for High or Very High Risk Prostate Cancer: European Validation of the Current NCCN(R) Guideline. The Journal of urology. 2017; 198: 354-61.

21. Sundi D, Tosoian JJ, Nyame YA, Alam R, Achim M, Reichard CA, et al. Outcomes of very high-risk prostate cancer after radical prostatectomy: Validation study from 3 centers. Cancer. 2019; 125: 391-7.

22. Reichard CA, Hoffman KE, Tang C, Williams SB, Allen PK, Achim MF, et al. Radical prostatectomy or radiotherapy for high- and very high-risk prostate 
cancer: a multidisciplinary prostate cancer clinic experience of patients eligible for either treatment. BJU international. 2019; 124: 811-9.

23. Zhu X, Albertsen PC, Andriole GL, Roobol MJ, Schroder FH, Vickers AJ. Risk-based prostate cancer screening. European urology. 2012; 61: 652-61.

24. Petrelli F, Vavassori I, Coinu A, Borgonovo K, Sarti E, Barni S. Radical prostatectomy or radiotherapy in high-risk prostate cancer: a systematic review and metaanalysis. Clinical genitourinary cancer. 2014; 12: 215-24.

25. Wallis CJD, Saskin R, Choo R, Herschorn S, Kodama RT, Satkunasivam R, et al. Surgery Versus Radiotherapy for Clinically-localized Prostate Cancer: A Systematic Review and Meta-analysis. European urology. 2016; 70: 21-30. 